\title{
Design and Implementation of a Computerized Management System for Adverse Drug Reactions at the Sourô SANOU University Hospital (CHUSS) in Bobo-Dioulasso, Burkina Faso
}

\author{
Arsene Sie Hamed TRAORE ${ }^{\mathrm{a}}$, Seydou Golo BARRO ${ }^{\mathrm{a}, \mathrm{b}, 1}$, Souleymane FOFANA ${ }^{\mathrm{a}}$, \\ Issiaka SOMBIE ${ }^{\mathrm{a}, \mathrm{c}}$, Rasmané SEMDE ${ }^{\mathrm{d}}$, Carine DE VRIESE ${ }^{\mathrm{e}}$, Dieudonne ILBOUDO ${ }^{\mathrm{a}}$ \\ and Pascal STACCINI ${ }^{\mathrm{b}}$ \\ ${ }^{a}$ Nazi BONI University, Bobo Dioulasso, Burkina Faso \\ ${ }^{\mathrm{b}}$ RETINES Lab - Côte d'Azur University, Nice, France \\ ${ }^{\mathrm{c}}$ West African Health Organization, Bobo-Dioulasso, Burkina Faso \\ ${ }^{d}$ Drug Development Laboratory, Joseph Ki-Zerbo University, Burkina Faso \\ e Pharmacology Department, Université Libre de Bruxelles (ULB), Belgique
}

\begin{abstract}
Pharmacovigilance is the science and activities related to the detection, evaluation, understanding and prevention of adverse drug reactions or any other possible drug-related problems. In our tropics, this discipline is in an embryonic state. The availability of a management system capable of responding to pharmacovigilance activities is the main objective of our study. The coding was done on the DJANGO Framework. Signal detection was done using the ROR method. We designed three modules which are the notification module, the analysis module and the statistics module. This study has allowed us to launch the basis for a computerization of the pharmacovigilance information system and partly meets our objective. However, it could lead to the integration of the dictionary of adverse effects such as MedDRA as well as the International Classification of Medicines (ATC, EphMRA).
\end{abstract}

Keywords. Pharmacovigilance, Signal detection, Adverse reactions

\section{Introduction}

The development of pharmacovigilance in the world is quite recent and was accentuated following the occurrence of various health scandals in the 19th century, the most notable of which was the thalidomide scandal [1]. In 2002, the World Health Organization defined pharmacovigilance as the science and set of activities related to the detection, evaluation, understanding and prevention of adverse reactions or any other drug-related problem [2]. Indeed, all health products put on the market have benefits and risks, as clinical trials do not provide all the information on the safety of a drug at the time of marketing, as some adverse reactions (ARs) only appear during the use of the drugs in

\footnotetext{
${ }^{1}$ Corresponding Author, Seydou Golo BARRO; E-mail: seydou.golo@gmail.com.
} 
the general population. This is the importance of pharmacovigilance, whose mission can be summarized around three actions: identification of AEs, which is based on reporting by health professionals, evaluation, which is based on imputability research, and prevention [3].

These three actions require the implementation of an efficient pharmacovigilance system. The first WHO collaborating center was created in Uppsala, Sweden (UMC) in 1978 [2]. Morocco and South Africa became the first African collaborating countries in 1992 [4]. The pharmacovigilance system in African countries is essentially characterized by spontaneous reporting, the inadequacy of local databases and the absence of tools for automatic generation of pharmacovigilance signals [4].

Burkina Faso is no exception to this observation, until 2013 the Sourô SANOU University Hospital (CHUSS) of Bobo-Dioulasso was the only hospital center to dedicate a service to pharmacovigilance [5]. This service is responsible for the detection, notification, analysis of adverse drug events and the use of this information for prevention purposes. In this service, spontaneous reporting is the main method of collection and individual analysis of imputability is done on a weekly basis.

A few isolated studies have consisted in evaluating pharmacovigilance signals in the department. However, the availability of a computerized management system for pharmacovigilance observations, which is able to carry out an imputability analysis and to generate signals automatically, is now essential. This has the advantage of improving the pharmacovigilance information system and making decisions that best meet patient safety in the use of medicines.

This is the general objective of our study, which consists of setting up a computerized management system for drug side effects at the Sourô SANOU University Hospital in Bobo-Dioulasso.

\section{Materials and Methods}

The study on the implementation of an automated management system of EIs was carried out at the CHUSS of Bobo Dioulasso which is a hospital of last resort in the health pyramid of Burkina Faso. At the server level, the programming language used is PYTHON 3.9. The HTML5, Javascripts and CSS3 languages were used to display, style and make dynamic the web elements of our pages. In order to avoid repetition of identical procedures during the creation of the web solution, we opted for the DJANGO Framework which is developed in python and responds to the DRY slogan " Don't Repeat Yourself ". This framework is open source and is dedicated to web development. Moreover, it uses an $\mathrm{MVT}^{2}$ model which is inspired by the $\mathrm{MVC}^{3}$ model. For the web server we used Apache 2.0. However, as almost all web servers do not communicate natively with python, we needed an interface to ensure this communication and DJANGO supports two interfaces which are WSGI ${ }^{4}$ and the new standard ASGI ${ }^{5}$. We used as database management system, MySQL version 5.7. The analysis and design method used is 2TUP, the modeling language is UML. For the drug accountability analysis we used the WHO algorithmic method and the French method updated by

\footnotetext{
${ }^{2}$ Model View Template

${ }^{3}$ Model View Controller

${ }^{4}$ Web Server Gateway Interface

${ }^{5}$ Asynchronous Server Gateway Interface
} 
Arimone et al. in 2011[6,7]. Signal detection was done using the Reporting odds Ratio [8]. Its formula is as follow:

$$
\begin{aligned}
& \left(\frac{a}{b}\right)+\left(\frac{c}{d}\right)=R O R \\
& R O R * e^{( \pm)^{1.96} \sqrt{\frac{1}{a}+\frac{1}{b}+\frac{1}{c}+\frac{1}{d}}}=I C
\end{aligned}
$$

Table 1: ROR formula coefficients

\begin{tabular}{lcc}
\hline Cases on drugs of interest & $\begin{array}{c}\text { Report on events } \\
\text { of interest }\end{array}$ & $\begin{array}{c}\text { Report on others } \\
\text { events }\end{array}$ \\
\hline Cases on the drug of interest & $\mathrm{a}$ & $\mathrm{b}$ \\
Cases on other drugs & $\mathrm{c}$ & $\mathrm{d}$ \\
Total & $\mathrm{a}+\mathrm{c}=\mathrm{N} 1$ & $\mathrm{~b}+\mathrm{d}=\mathrm{N} 2$ \\
\hline
\end{tabular}

\section{Results}

The designed solution is a web application which is then independent of operating systems and therefore accessible from all devices. Figure 1 displays the home interface of the solution.

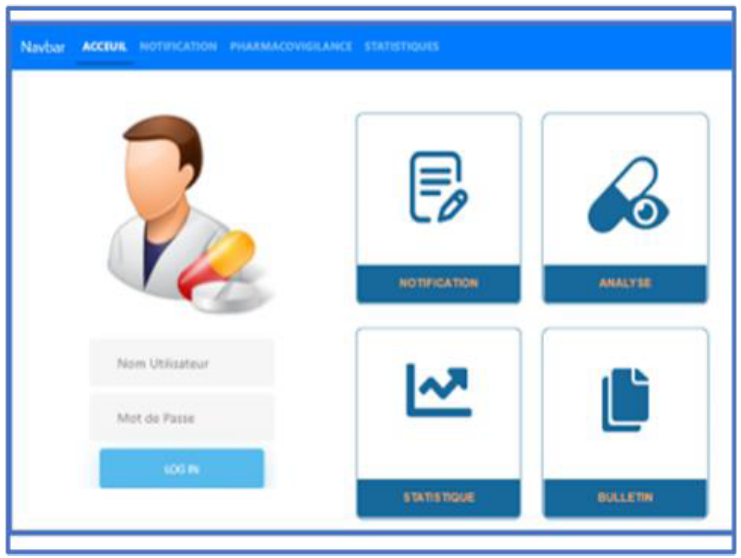

Figure 1. Welcome interface

Also the functionalities of our solution are divided into modules in order to facilitate the maintainability and to ensure an ergonomics live up to your expactations of all the users. The system includes essentially three modules which are:

- Notification management module that allows users to record information on the patient, on the drugs consumed, on the prescriber and on the adverse effects.

- Analysis module which allows the combination of each drug entered in the previous module with each of the reported adverse events. This module also allows an assignment of the imputability scores of the adverse effects.

- Statistics management module allowing to generate the signal for the drugs and the adverse reactions with the highest ORR scores. This module allows to elaborate dashboards for a decision support at the level of the 
pharmacovigilance service. It also provides the possibility of a personalized search.

The access to the software is conditioned by authentication and the access to the different modules is conditioned by the authorization levels of the different actors and users of the system.

\section{Discussion}

Our objectives were to make available a database capable of responding in real time to the recording, analysis and restitution of data from the pharmacovigilance service. Our results show that these objectives have been achieved because we have developed an application that takes into account: the modules of recording, management and analysis of adverse drug reactions. The evaluation of our system is in progress through the compilation of the paper files already collected on the years spent in the pharmacovigilance service. The implementation of our system is a great innovation in the field of pharmacovigilance in our country and in the African sub-region, although it needs improvement, which are the next steps of this work. These are the integration of the dictionary of adverse reactions such as MedDRA, the International Classification of Medicines (ATC, EphMRA) etc. Another challenge remains the mobilization of ressources for deployment of the system on the system on a remote server to allow its use by other structures in Burkina Faso and even in the sub region.

\section{References}

[1] Lefrère J-J, Berche P. Les bébés du thalidomide. Presse Médicale. 2011;40:301-308.

[2] Weltgesundheitsorganisation, Collaborating Centre for International Drug Monitoring, editors. The importance of pharmacovigilance: safety monitoring of medicinal products. Geneva: WHO [u.a.]; 2002.

[3] The Importance of Pharmacovigilance - 2002 (WHO) - PAHO/WHO | Pan American Health Organization [Internet]. [cited 2020 May 15]. Available from: https://www.paho.org/en/documents/importance-pharmacovigilance-2002-who.

[4] Isah AO, Pal SN, Olsson S, et al. Specific features of medicines safety and pharmacovigilance in Africa. Ther Adv Drug Saf. 2012;3:25-34.

[5] Kabore L, Millet P, Fofana S, et al. Pharmacovigilance Systems in Developing Countries: An Evaluative Case Study in Burkina Faso. Drug Saf. 2013;36:349-358.

[6] Arimone Y, Bidault I, Dutertre J-P, et al. Réactualisation de la méthode française d'imputabilité des effets indésirables des médicaments. Therapies. 2011;66:517-525.

[7] Bannwarth B. Critères d'imputabilité des effets indésirables des médicaments. Rev Rhum Monogr. 2010;77:173-175.

[8] Faillie J-L. Les études cas-non cas : principe, méthodes, biais et interprétations. Thérapie. 2018;73:247255. 